\section{Community-associated Staphylococcus aureus infections: pneumonia}

\author{
Marios Karvouniaris, \\ Demosthenes Makris, \\ Epameinondas Zakynthinos
}

Critical Care Medicine Department, University Hospital of Larissa, Greece

\begin{abstract}
Community-associated methicillin-resistant Staphylococcus aureus (CA-MRSA) is an emerging health problem with distinct epidemiology. CA-MRSA colonization and infection is associated with risk factors different from healthcare-associated methicillin-resistant $S$. aureus infection. CA-MRSA strains present different characteristics to healthcare associated strains in terms of microbiology as well. Moreover, infection as a result of CAMRSA may be associated with severe infections, in particular necrotizing pneumonia. CAMRSA strains may produce Panton-Valentine leukocidin, a protein that available data suggest to be associated with the severity of the infection. Although the incidence of CA-MRSA pneumonia is relatively low, it affects mostly young, immunocompetent individuals, and in this respect constitutes a serious and potentially lethal form of community-acquired pneumonia. Current treatment suggested by international consensus guidelines includes linezolid or vancomycin often combined with clindamycin and/or rifampicin. However, clinical studies are required to clarify further therapeutic issues on timing, dosing, and choice of optimum treatment, and whether new therapeutic strategies such as vaccination and immunoglobulins could be useful. In the present review we discuss the microbiology, epidemiology, pathogenesis, and clinical aspects of community-acquired pneumonia as a result of CA-MRSA in respect of management and prevention.
\end{abstract}

\section{Introduction}

During the last ten years an old pathogen, Staphylococcus aureus, has created new public health problems. Methicillin-resistant $S$. aureus (MRSA) is the cause of emerging infections in the community, a result of strains that differ from the usual healthcare-associated ones and are referred to as community-associated MRSA (CA-MRSA) strains. Based on recent definitions, ${ }^{1}$ diagnosis of infection because of CA-
MRSA requires the following epidemiological criteria: i) the diagnosis was made in the outpatient setting or by a culture positive for MRSA within 48 hours after admission to hospital; ii) no medical history of colonization or infection with MRSA; iii) absence of hospitalization, admission to a nursing home, skilled nursing facility or hospice, dialysis, or surgery in the past year; iv) absence of permanent indwelling catheters or medical devices that pass through the skin into the body.

Currently, MRSA infection is considered a major public health issue because it affects young, immunocompetent individuals. CAMRSA has the potential to cause severe skin and soft tissue infections and most notably, pneumonia (CAP-MRSA), which can be severe and necrotizing. Thus, CA-MRSA is associated with considerable morbidity and even mortality in the case of necrotizing CAP-MRSA. However, CAP-MRSA is a relatively novel entity and there are still unanswered questions in several aspects of CAP-MRSA.

In the present review we discuss the microbiology, epidemiology, pathogenesis and clinical aspects of CAP-MRSA in respect of its management and prevention.

\section{Microbiology}

CAP-MRSA is caused by communityacquired staphylococcus strains. These are ubiquitous bacteria and morphologically grampositive Staphylococcus cocci. ${ }^{2}$ Their main representative, $S$. aureus subsp. aureus, is highly resistant to extreme and adverse environmental conditions, such as drying as well as high $\mathrm{NaCl}$ concentrations, ${ }^{3}$ and can grow at a temperature range of 15 to 45 degrees. ${ }^{4}$ This explains its ability to colonize the nasal mucosa especially but also the pharyngeal mucosa and the skin (usually of the perineum and the axillae) of $50 \%$ of the general population. Thus, a high percentage of the population may chronically carry the microbe for years and such individuals are at increased and continuous risk of subsequent infection., ${ }^{5,6}$ Moreover, $S$. aureus strains produce a number of extracellular enzymes (such as coagulase, hyaluronidase, penicillinase) and toxins (hemolysins, staphylococcal superantigens, and leukocidins), which function as virulence factors. In addition, $S$. aureus strains produce catalase, which protects them from hydrogen peroxide produced by the phagocyte.

The main mechanism of resistance to methicillin (MRSA strains) is not dependent on penicillinase, but on the production by $S$. aureus of a different penicillin-binding protein, PBP2A or PBP2', with low-binding affinity to $\beta$-lactams. This different protein is encoded by the mecA gene, which is situated on the
Correspondence: Demosthenes Makris, Lecturer of Critical Care Medicine, ICU, University Hospital of Larissa, Greece. E-mail: appollon7@hotmail.com; dimomakris@med.uth.gr

Key words: staphylococcal pneumonia, community-acquired pneumonia, resistance, community-associated

Staphylococcus aureus infections.

Contributions: MK drafted the manuscript, DM reviewed the paper for important intellectual content, and EZ motivated the study.

Conflict of interest: the authors report no conflicts of interest.

Received for publication: 2 April 2010.

Revision received: 17 June 2010.

Accepted for publication: 10 July 2010.

This work is licensed under a Creative Commons Attribution 3.0 License (by-nc 3.0).

(C) Copyright M. Karvouniaris et al., 2010

Microbiology Research 2010; 1:e4

doi:10.4081/mr.2010.e4

staphylococcal cassette chromosome mec (SCCmec), an element of extra-chromosomal DNA. SCCmec has been characterized as a novel, mobile resistance element that differs from both transposons and bacteriophages. ${ }^{8}$ To date, five SCCmec types (I-V) have been distinguished. ${ }^{9}$ All SCCmec elements carry the gene mecA, which has identical nucleotide content irrespective of the SCCmec type and the methicillin MICs of the MRSA strains that may range from $<2 \mathrm{mg} / \mathrm{L}$ to $>1000 \mathrm{mg} / \mathrm{L}^{10}$ The gene mecA codes for the penicillin-binding protein PBP2a, which allows for the synthesis of the peptidoglycan layer and cell wall synthesis in the presence of $\beta$-lactams. ${ }^{9}$

Thus, the study of the epidemiology of MRSA strains is based on screening for SCCmec, the meqA gene, and the genes lukF and $l u k S$ by polymerase chain reaction (PCR). For epidemiological reasons as well, screening of the MRSA genotype is done with pulsed-field gel electrophoresis. The genes $l u k F$ and $l u k S$ encode Panton-Valentine leukocidin (PVL) and are present in the majority of CA-MRSA strains (overall in 77\%). The PVL locus is situated in a bacteriophage that occurs in less than $5 \%$ of $S$. aureus strains worldwide. In addition to PVL, CA-MRSA strains are characterized by increased prevalence of other toxins ( $\alpha$-toxin and staphylococcal enterotoxin B). ${ }^{11}$ Hence, these strains are considered rather more virulent than the hospital-acquired MRSA strains, and in this respect CAP-MRSA may present necrotizing characteristics and may be associated with an adverse outcome. Furthermore, unlike healthcare-associated methicillinresistant $S$. aureus strains that gradually develop resistance to other antibiotic classes in 
addition to all $\beta$-lactams (penicillins, cephalosporins, carbapenems) resistance, $\mathrm{CA}$ MRSA resistance is confined mostly to $\beta$-lactam antibiotics (Table 1). ${ }^{12}$ In this respect, epidemiological criteria have been established that distinguish CA-MRSA infection, and therefore CAP-MRSA, from infection as a result of healthcare-associated MRSA infection (see definition).

\section{Epidemiology}

It has been about 50 years since hospital strains of MRSA were first described. ${ }^{12}$ However, in parallel with the presence of healthcare-associated MRSA, a new family of strains that caused infections in the community (CA-MRSA), including younger and healthier persons, without risk factors for the development of MRSA infection, has appeared. CA-MRSA strains have spread to the general population and MRSA is identified very often as a cause of skin and soft-tissue infections among patients presenting to emergency departments in the United States and Greece as well. ${ }^{13,14}$ CAP-MRSA is commonly encountered during the influenza season and usually occurs in children, adolescents, and young adults without underlying diseases (lung or hematologic disease, immunosuppression, diabetes mellitus). Previous studies have depicted risk factors for colonization with CAMRSA and development of infections. ${ }^{15}$ Clinically, CA-MRSA strains are capable of causing serious invasive disease that is sometimes fatal. ${ }^{16}$ In a recent series of 51 patients from the United States with staphylococcal pneumonia in the community, $73 \%$ of the isolates were MRSA and only half of the patients have been given appropriate antibiotic therapy (linezolid or vancomycin). ${ }^{17}$

CA-MRSA strains are spreading to the hospitals as well and are replacing ${ }^{18}$ the traditional HA-MRSA strains. Thus, there is also an emergence of the staphylococcal cassette chromosome mec type IV methicillin-resistant $S$. aureus as a cause of ventilator-associated pneumonia (VAP, pneumonia presented after 48 hours of endotracheal intubation). These CA-MRSA strains were responsible for $20 \%$ of the total MRSA-VAP cases in an academic institution. These were usually PVL-positive strains. In these strains, the presence of leukocidin was not associated with necrotizing pneumonia. ${ }^{19} \mathrm{CA}$-MRSA caused $<1 \%$ of cases of CAP admitted to the hospital according to a recent retrospective study.

\section{Pathogenesis}

S. aureus infections are caused by strains that have already colonized nasal mucosa making the colonized persons a reservoir for the spread of the microbe. ${ }^{21}$ Pneumonia results (55\% of cases) mainly from aspiration of nasooropharyngeal secretions colonized by $S$. aureus, which usually occurs after infection with the influenza virus. The rest of the cases can occur by hematogenous dissemination, secondary to the infection of skin or soft tissues, as well as after septic abortion. The presentation is typically severe regardless of the previous health status of the patient. ${ }^{22}$

In the case of CA-MRSA, however, a few striking differences compared to other MRSA and MSSA infections should be noted. In CAMRSA cases, non-nasal colonization predominates in axillae, perineum, and rectum (the latter explaining the sexual transmission of the pathogen). In addition, in outbreaks CAMRSA infection was associated with exposures to various contaminated fomites shared by athletes, relatives, or roommates, including whirlpools, shared razors, and shared towels. ${ }^{23}$ These characteristics of MRSA pathogenesis of infection should be kept in mind.

It has also been suggested that respiratory viral infections make the bronchial surface more vulnerable to secondary bacterial infection by facilitating the attachment of bacteria, such as staphylococci, on the destroyed respiratory epithelia, allowing the aspiration of oropharyngeal contents contaminated with $S$. aureus. Thus, previous studies found a positive association between influenza infection and staphylococcal pneumonia. This relationship might also be present between CAP-MRSA and the influenza virus. However, available epidemiological studies are few and results are not consistent. In a recent study that included cases with CAP-MRSA, $33 \%$ of those examined were positive for the influenza virus. However, a rapid influenza test with low sensitivity was used and thus one might argue that this incidence rate might underestimate the actual number of subjects with influenza. In addition, the presence of other respiratory viruses which might also favor bacterial infections have not been assessed. ${ }^{17}$ On the other hand, in a recent study from Australia ${ }^{24}$ that examined co-infection with the new pandemic H1N1 influenza virus and CAP-MRSA, there were only five co-infections out of $252 \mathrm{H} 1 \mathrm{~N} 1$ cases.

In addition, it has been suggested that the presence of a preceding influenza infection might be associated with the necrotizing character of CAP-MRSA. Thus, patients with ventilator-associated pneumonia because of CAMRSA who did not present a preceding influenza infection, did not develop a necrotizing infection as well. ${ }^{19}$ However, as it was pointed out, more studies are required to clarify the potential contribution of a respiratory virus infection in the course of CAP-MRSA.

Another important issue in the pathogenesis of CA-MRSA infections that has been highlighted previously is the role of an extracellular toxin (leukocidin) described by Panton-Valentine. PVL was first described in 1932 and is also referred to in the literature with the abbreviations Luk-PV. ${ }^{3}$ The presence of PVL has been considered to correlate with increased severity of infections as a result of $S$. aureus. ${ }^{3}$ Apart from CAP-MRSA, these can be

Table 1. Main characteristics of HA-MRSA and CA-MRSA strains.

\begin{tabular}{lll} 
Characteristic & HA-MRSA & CA-MRSA \\
Origin & Hospital, hospice etc. & Community \\
Infections & $\begin{array}{l}\text { Surgical site infections, line- or device-associated } \\
\text { infections, ventilator-associated pneumonia }\end{array}$ & $\begin{array}{l}\text { Skin and soft tissue infections, necrotizing community-acquired } \\
\text { pneumonia, necrotizing fasciitis }\end{array}$ \\
\hline Epidemiology & Hospitalized, elderly & Young, athletes, injection drug users, military personnel \\
Antimicrobial resistance & Multidrug resistance & Mainly $\beta$-lactams \\
\hline PVL & Absent & Usually present \\
Methicillin resistance elements & SCCmeq I-III & SCCmeq IV-V \\
\hline Genotype & USA 100 & USA 300 \\
Active antibiotics & Vancomycin, linezolid, daptomycin & $\begin{array}{l}\text { Vancomycin, linezolid, daptomycin trimethoprim/sulphomethoxazole, } \\
\text { doxicycline, clindamycin }\end{array}$ \\
\hline
\end{tabular}

HA-MRSA, hospital-acquired methicillin-resistant Staphylococcus aureus; CA-MRSA, community-associated methicillin-resistant Staphylococcus aureus. 
skin and soft tissue infections that need surgical debridement and septic, potentially fatal infections such as osteomyelitis, necrotizing fasciitis, and the Waterhouse-Friderichsen syndrome. ${ }^{12}$ The severity of these infections and thus of CAP-MRSA has been attributed to the varying leukocytolytic, erythrocytolytic, and dermonecrotic properties of the toxin. The toxin forms heptameric pores in the leukocyte membrane and destroys the leukocyte, with the release of inflammatory enzymes and cytokines. ${ }^{3}$ At lower concentrations it induces the apoptosis of leukocytes. ${ }^{25}$ In a mouse staphylococcal pneumonia model only PVLpositive (PVL+) strains caused necrotizing pneumonia. ${ }^{26}$ Moreover, PVL acts differentially on neutrophils of various species, which suggests that PVL has an important cytotoxic role in human neutrophils. This has major implications for the pathogenesis of CA-MRSA infections. ${ }^{27}$ Based on this data, the role of PVL for the severity of infections is potentially important. However, we should also underline here that severe CAMRSA infections may be present event in the absence of PVL, as it was demonstrated in studies with PVL-strains. ${ }^{28}$ It is therefore likely that not only PVL but also other microbial or host factors might contribute to the severity of CAP-MRSA. ${ }^{29}$

\section{Clinical Presentation}

On the whole, the clinical presentation of CAP-MRSA is one of a septic syndrome, and if it begins as influenza it continuously deteriorates and rapidly progresses to pneumonia, which is sometimes fatal. The presentation of the disease is unique and not that of a biphasic illness (influenza-like illness and then pneumonia) as it was formerly described. ${ }^{17}$ In the majority of cases, the disease is characterized by symptoms of the respiratory system - of the influenza type - that either precede or coexist with pneumonia, such as fever, cough, or sore throat. Fever is usually high $\left(>39^{\circ} \mathrm{C}\right)$. Symptoms indicating involvement of the lower respiratory system such as tachypnea ( $>30$ breaths per minute), cyanosis, and cough are common. The cough is often productive and it is more likely for hemoptysis than for purulent sputum to be present. A common complication of CAP-MRSA is empyema. General symptoms including circulatory signs and symptoms of encephalopathy are also commonly observed especially in more severe disease. Tachycardia, often $>140$ beats per minute, and hypotension indicate risk of circulatory collapse. Symptoms of encephalopathy such as lethargy, confusion, or even coma are commonly observed during the course of the disease. Sometimes rash, boils, or diarrhea are also observed. . $^{170-33}$
The most characteristic laboratory finding is leukopenia although leukocytosis is more commonly found. Chest X-ray findings are usually bilateral with rather symmetric consolidation. Cavitations, nodules, or pleural effusions are not often found. In subsequent chest X-ray examinations the areas of consolidation become progressively confluent. ${ }^{34}$ The most common chest computer tomography (CT) scan findings are bilateral, rather symmetricconsolidation as well as bilateral, rather ground-glass opacities, centrilobular nodules, and cavitations (either in regions of consolidation or in large nodules). Pleural effusions and mediastinal lymphadenopathy are found in the majority of cases. ${ }^{34}$

Examination of necrotic materials in patients with necrotic PVL-positive pneumonias has shown tracheal and bronchial mucosal ulceration with a gray-white necrotic appearance. Abundant hemorrhagic secretions were found in the main bronchi while the lungs were solidified, congestive, and hemorrhagic. Microscopic examination revealed infiltration of the respiratory epithelium by inflammatory cells, and ulcerations coated by extensive gram-positive cocci colonies throughout the respiratory tract (larynx to lobar bronchi). The lung parenchyma was characterized by alveolar hemorrhage, necrosis of the interalveolar septa, and large clusters of gram-positive cocci. ${ }^{30}$

\section{Diagnosis}

Diagnosis of CAP-MRSA might be difficult owing to the fact that CA-MRSA infections have not been considered as likely in the past. The fact that only half of the 51 US patients with staphylococcal pneumonia in the community were given appropriate antibiotic therapy (73\% of the isolates were CA-MRSA) $)^{17}$ and, in addition, the potential fulminant course of CAPMRSA underlined the necessity to clearly define CAP-MRSA and to achieve prompt diagnosis and therefore management. According to the guidelines for the community-acquired pneumonia in adults ${ }^{35}$ CAP-MRSA should be considered in cases with pneumonia when: i) influenza-like illness preceded, and ii) cavitations are found in chest X-ray without risk factors for the development of aspiration pneumonia owing to anaerobic microbes. Other authors ${ }^{15,36,37}$ point out that the clinical diagnosis of CAP-MRSA should be considered when influenza-like illness precedes and the following factors are present: young patients with hemoptysis, respiratory failure, tachypnea of >30 breaths/min, high fever, leukopenia, hypotension, tachycardia of $>140$ beats/min, and pneumatoceles, abscesses, or multilobar involvement in the chest X-ray. In general, CAP-
MRSA should be considered in subjects with risk factors for infection or known to be colonized by CA-MRSA when clinical symptoms indicate acute gram-positive coccial pneumonia.

The finding of clusters of gram-positive cocci in bronchoalveolar lavage (BAL) fluid, a tracheal aspirate sample, in proper sputum samples, or in pleural fluid may be diagnostic. Gram staining in blood samples might also indicate contamination. ${ }^{35,36}$ Thus, in a retrospective analysis that included patients with CAP-MRSA, ${ }^{20} 73 \%$ of them had positive cultures in specimens obtained from the respiratory track (BAL, bronchial secretions, sputum) $33.3 \%$ had positive pleural fluid cultures while $33 \%$ had positive blood cultures. In this respect, it has been suggested that negative gram stain and culture results of an adequate sputum specimen should be sufficient to withhold or stop treatment for MRSA infection. ${ }^{35}$

On the other hand, recently developed molecular methods ${ }^{38}$ have the potential to improve diagnosis further. Molecular methods are rapid diagnostic tests that reduce the time of detection of MRSA from days to hours. Many tests have high sensitivity and specificity that exceeds $90 \%{ }^{38}$ Recently molecular methods ${ }^{39}$ have been validated and were highly accurate (with a sensitivity and specificity that are close to $100 \%$ ) in the detection of MRSA in clinical samples, such as blood, pus, wounds, cerebrospinal fluid, and urine. These methods can also give useful information for the presence of PVL protein. ${ }^{39}$ However, the authors have not tested sputum samples. In addition, the diagnostic yield of PCR techniques in CAPMRSA is not yet known as there is a lack of studies in this field, and thus this is an issue that offers the opportunity to be explored in the future.

\section{Prognosis}

CAP-MRSA is a potentially fatal infection and ICU admission was necessary in the vast majority (67-81\%) of patients in previous studies. Among those admitted in the ICU more than $50 \%$ needed intubation and mechanical ventilation while their stay in ICU was often prolonged. Mortality in these populations is high (29-60\%), depending on the study. ${ }^{17,31,33}$

The poor prognosis of CAP-MRSA is linked to severity factors of pneumonia such as the development of adult respiratory distress syndrome (ARDS) or the need for inotropes or mechanical ventilation. The presence of hemoptysis (which likely reflects necrosis of the respiratory mucosa) is associated with rapid death. In a multivariate analysis, leukopenia and erythroderma (not associated with toxic shock syndrome) occurring within the 
first 24 hours after admission to the hospital were independently associated with fatal outcome. $^{40}$ The presence of leukopenia $(<4000 / \mathrm{mL})$ has been pointed out by other authors as a significant independent risk factor as well. ${ }^{17}$ In the same study, ${ }^{17}$ co-infection with both influenza and Staphylococcus has been associated with a greater likelihood of death; however, this association did not remain significant in the multivariate analysis of data.

\section{Prevention}

Firstly, we should avoid the spread of $S$. aureus in the community by implementing the basic principles of hygiene and by the correct diagnosis and treatment of the infection that this pathogen causes (Table 2). ${ }^{41}$ Screening for nasal or skin carriers of the microbe is not necessary and decolonization should be avoided because of the fear of developing further antimicrobial resistance. Effectiveness of decolonization decreases after a few weeks, and concerning the pathogenesis of CA-MRSA (with the increased importance of non-nasal colonization), nasal mupirocin is not enough, making the undesirable use of systematic antibiotics necessary. ${ }^{5}$

A conjugated, bivalent vaccine against capsular antigens of $S$. aureus (StaphVAX), ${ }^{42}$ that can protect patients receiving hemodialysis from bacteremia and needs repeating the dose, was initially effective but a phase III trial failed to confirm the result. ${ }^{43} \mathrm{~A}$ phase II clinical trial for the prevention of $S$. aureus in elective cardiothoracic surgery patients is under way. A vaccine against the cell wall protein IsdB is being tested. ${ }^{43,44}$ In terms of prevention with the use of passive immunization, a human immunoglobulin-G (IgG) preparation known as INH-A21 that contains elevated levels of antibodies to the staphylococcal surface adhesins ClfA and SdrG was given (phase III) to neonates but it failed to show a clinical benefit. ${ }^{45}$ Staphylococcal infections have a complex pathogenesis through many toxins and enzymes. Thus, a multicomponent vaccine

Table 2. Potential strategies for preventing transmission of $S$. aureus in the community.

Compliance with hand hygiene

Keep draining wounds covered

Sharing of personal items (razors, towels) should be avoided

Avoid contact sports if a potential participant has an open skin lesion

Do not allow use of whirlpools or saunas to persons with open skin lesions

Make sure that communal bathing facilities are clean is needed and the component antigens have to be highly conserved, thus include both toxoids and surface antigens as well. Some candidate antigens are tested in pre-clinical trials. ${ }^{43,44}$ In two of them, mice were successfully vaccinated with $\alpha$-hemolysin and PVL. ${ }^{46,47}$

\section{Treatment}

The basic principles of CA-MRSA pneumonia treatment are not confined to antibiotic prescription (Table 3). It is advisable that the patients are admitted to the ICU with infectious disease consultation in order to make sure that the vital signs are properly supported and the patient is given the best possible antibiotic therapy. Despite the fact that sputum, pleural fluid, and blood cultures will support or confirm CAP-MRSA, their results should not delay treatment decisions, and thus patients should receive therapy as soon as possible, and in the case of empyema, the pleural cavity should be properly drained. ${ }^{35,36}$

As has been underlined, antibiotic therapy should be given early, while the patient is still in the outpatient department after blood, sputum, and/or pleural fluid cultures have been done. ${ }^{15,35,36}$ Secondly, treatment should include drugs that combine anti-staphylococcal action with anti-MRSA activity and suppress the production of PVL toxin. ${ }^{37}$ Unfortunately, there have been no well conducted randomized trials yet, especially for CAP-MRSA. Various combinations of vancomycin, clindamycin, linezolid, rifampicin, and cotrimoxazole have been used in various doses and with different success rates. ${ }^{15}$

The pharmacokinetic/pharmacodynamic $(\mathrm{pK} / \mathrm{Pd})$ properties of vancomycin include limited volume of distribution, inability of passively diffusing through plasmatic membrane, and water solubility. These properties may not be advantageous in treating pneumonia and, in this respect, the most severe forms of CAPMRSA especially. ${ }^{48}$ Vancomycin's average levels in lung epithelial lining fluid (ELF) are only $20 \%$ of blood levels and the recommended dose of the antibiotic ( $1 \mathrm{~g}$ twice daily) is likely to be suboptimal for treating pneumonia. ${ }^{48}$ A clinical study of nosocomial MRSA pneumonia has shown that despite the appropriate regimen containing glycopeptides (vancomycin or teicoplanin), increased mortality was shown, apart from patients who received vancomycin by continuous intravenous infusion and had a better prognosis. ${ }^{49}$ Therefore, for the treatment of MRSA pneumonia, plasma trough levels should be maintained higher than currently recommended (usually $15-20 \mathrm{mg} / \mathrm{L}$ and even $30-35 \mathrm{mg} / \mathrm{L}$ instead of $10-15 \mathrm{mg} / \mathrm{L}$, with the risk of developing complications) and may be given by continuous infusion. Thus, it has been suggested $^{48}$ that vancomycin should start with a loading dose of $15 \mathrm{mg} / \mathrm{kg}$ followed by a continuous infusion of $30 \mathrm{mg} / \mathrm{kg} / \mathrm{day}$, and to add rifampicin as well.

In addition, MRSA strains with MIC levels for vancomycin equal to or less than $1 \mathrm{mg} / \mathrm{L}$ indicate increased risk of treatment failure. Furthermore, heteroresistance might be developed where, despite the apparent susceptibility, there are subpopulations of bacteria with resistance to the drug. ${ }^{50}$ In addition, vancomycin does not have any effect on toxin levels and the inappropriate administration of nafcillin ( $\beta$-lactam) may increase PVL production. ${ }^{11}$ These considerations limit the usefulness of vancomycin in the setting of CAPMRSA and suggest that vancomycin may have to be replaced with other agents with adequate anti-MRSA activity and suppression of the production of PVL toxin such as linezolid or clindamycin..$^{51}$

On the contrary, linezolid may dramatically reduce PVL levels through inhibition of protein synthesis. ${ }^{11}$ Linezolid is a moderately lipophilic compound and presents $\mathrm{pK} / \mathrm{pD}$ properties such as large volume of distribution, ability to diffuse easily through plasmatic membranes, ${ }^{48}$ and at this point offers advantages in treating serious lung infections. It has shown drug ELFto-plasma ratios on average $>2$ at different time intervals after administration. ${ }^{48}$ Moreover, resistance to linezolid is extremely rare. ${ }^{52} \mathrm{~A}$ previous study of nosocomial MRSA pneumonia showed that linezolid was associated with significantly better survival and clinical cure
Table 3. Therapeutic principles for CAP-MRSA.

\begin{tabular}{ll} 
Antimicrobials & Other measures \\
Linezolid $600 \mathrm{mg}$ twice daily & Cultures (blood, pleural fluid, sputum) \\
Vancomycin $1 \mathrm{~g}$ twice daily & Admission to the ICU \\
\hline Clindamycin $1.2-1.8$ g every six hours & Infectious disease physician consultation \\
Rifampicin $600 \mathrm{mg}$ twice daily & Infection control measures \\
\hline Linezolid* +clindamycin* ${ }^{ \pm}$rifampicin* combination & Pleural drainage in case of empyema \\
\hline CAP-MRSA, community-associated pneumonia methicillin-resistant Staphylococcus aureus; ${ }^{*}$ doses as described in the table.
\end{tabular}


rates than vancomycin. ${ }^{53}$ In another study, ${ }^{54}$ despite CAP-MRSA patients having received appropriate treatment with vancomycin, they presented clinical deterioration. Vancomycin was therefore changed with success to linezolid plus rifampicin or clindamycin.

The ATS Consensus Statement on the Management of CAP in Adults ${ }^{35}$ states that when suspecting CAP-MRSA, linezolid or vancomycin should be added to the basic regime. The need to administer linezolid or clindamycin for decreasing PVL production is also emphasized although the best possible therapy has not yet been defined because of the lack of clinical studies.

Other agents such as rifampicin ${ }^{51}$ have been used for treating CA-MRSA infections as they have excellent in vitro activity against CAMRSA. Moreover, the pharmacokinetic profile of rifampicin includes high lipid solubility and a large volume of distribution. ${ }^{55}$ Thus, some authors have suggested the routine addition of rifampicin $600 \mathrm{mg}$ twice daily if PVL+ staphylococcal pneumonia is suspected. ${ }^{15}$ However, rifampicin cannot be used as a single agent because of the rapid emergence of resistance but rather used in combination with other agents. Before such a decision, the interaction of rifampicin with other agents should be considered. For instance, combinations of rifampicin with trimethoprim/sulfomethoxazole (TMP/SMX) have been shown to exhibit antagonism in vitro. ${ }^{51}$ Many isolated CA-MRSA strains present susceptibility to TMP/SMX in vitro. ${ }^{13,14}$ However, despite its good in vitro activity TMP/SMX has been associated with clinical failures when a high bacterial burden or small abscesses exist. For example, in the case of a lung abscess, TMP/SMX treatment was associated with a higher rate of failures compared to vancomycin. ${ }^{56}$

Clindamycin can work in an environment with a high bacterial burden, has anti-PVL activity, and is cheap. ${ }^{57}$ Clindamycin is lipophilic, has good intracellular penetration, ${ }^{58}$ and in this respect its use offers advantages in the treatment of pneumonia and empyema. ${ }^{57}$ However, it exhibits inducible resistance during therapy and therapeutic failures have been observed. This phenomenon can be easily diagnosed with the D-zone test that discrim inates the fully sensitive strains from the ones that show inducible resistance. Thus, recent guidelines for UK practice ${ }^{15}$ recommended the use of high-dose clindamycin 1.2-1.8 g six hourly in the initial empiric treatment; as an alternative to add linezolid $600 \mathrm{mg} 12$ hourly. The duration of therapy is not defined.

Despite covering MRSA, daptomycin is inhibited by surfactant in vitro and is therefore not suitable for treating pneumonia. ${ }^{59}$ The role of tigecycline, ceftobiprole (cephalosporin with anti-MRSA activity), novel glycopeptides (dalbavancin, telavancin, oritavancin), and iclaprim has not been defined for the treat- ment of CA-MRSA pneumonia. ${ }^{50}$

\section{Adjunctive therapies}

A hyperimmune polyclonal antibody preparation (Altastaph) derived from healthy volunteers immunized with StaphVAX was test$\mathrm{ed}^{60}$ in patients with $S$. aureus bacteremia and was ineffective.

Immunoglobulin infusion provides high concentrations of anti-PVL antibodies, and at least in vitro, neutralizes the toxin. ${ }^{6}$ Immunoglobulin should thus be considered in severe sepsis and necrotizing pneumonia known or suspected to be because of $S$. aureus. The recommended dose is $2 \mathrm{~g} / \mathrm{kg}$ of body weight and is repeated at 48 hours if there is still evidence of sepsis or failure to respond. ${ }^{15}$ Activated protein $\mathrm{C}$ might be beneficial in very early sepsis in the setting of CAP-MRSA but once active hemorrhage has occurred the agent should not be used. ${ }^{15}$

\section{Conclusions}

CA-MRSA has emerged as a serious public health issue and is responsible for a severe form of community-acquired pneumonia. Despite the progress in our knowledge of the pathogen, more studies are needed to better clarify its pathogenesis and the role of its virulence factors, like PVL toxin. Wider public awareness of the problem and implementation of the basic principles of hygiene will help to prevent its spread. Finally, the rising microbial resistance and the fear of running out of antibiotics leads to research for effective anti-MRSA immunotherapy. The latter consists of antibody therapy and, hopefully, an effective vaccine might solve the problem in the long run.

\section{References}

1. CDC Department of Health and Human Services. Community-associated MRSA information for clinicians. Date last modified: February 3, 2005. Available from: http://www.cdc.gov.

2. Moreillon P, Que Y-A, Glauser MP. Staphylococcus aureus. In: Principles and practice of infectious diseases. 6th ed. Mandell GL, Bennett JE, Dolin R, eds. Philadelphia: Elsevier, 2005, pp 2321-51.

3. Matouskova I, Janout V. Current knowledge of methicillin-resistant Staphylococcus aureus and community-associated methicillin-resistant Staphylococcus aureus. Biomed Pap Med Fac Univ Palacky Olomouc Czech Repub 2008;152:191-202.

4. Todar K. Staphylococcus aureus and staphylococcal disease. In: Todar's Online
Textbook of Bacteriology. Available from: www.textbookofbacteriology.net/staph.

5. Ammerlaan HSM, Kluytmans JAJW, Wertheim HFL, et al. Eradication of methicillin-resistant Staphylococcus aureus carriage: A systematic review. Clin Infect Dis 2009;48:922-30.

6. Datta R, Huang SS. Risk of infection and death due to methicillin-resistant Staphylococcus aureus in long-term carriers. Clin Infect Dis 2008;47:176-81.

7. Mandell GL. Catalase, superoxide dismutase, and virulence of Staphylococcus aureus. In vitro and in vivo studies with emphasis on staphylococcal-leukocyte interaction. J Clin Invest 1975;55:561-6.

8. Appelbaum PC. Microbiology of antibiotic resistance in Staphylococcus aureus. Clin Infect Dis 2007;45:S165-70.

9. Deurenberg RH, Vink C, Kalenic S, et al. The molecular evolution of methicillinresistant Staphylococcus aureus. Clin Microbiol Infect 2007;13:222-35.

10. Giannouli S, Labrou M, Kyritsis A, et al. Detection of mutations in the FemXAB protein family in oxacillin-susceptible mecA-positive Staphylococcus aureus clinical isolates. J Antimicrob Chemother 2010;65:626-33.

11. Stevens DL, Ma Y, Salmi DB, et al. Impact of antibiotics on expression of virulence-associated exotoxin genes in methicillin-sensitive and methicillin-resistant Staphylococcus aureus. J Infect Dis 2007;195:202-11.

12. Bartlett J. Methicillin-resistant Staphylococcus aureus infections. Top HIV Med 2008;16:151-5.

13. Moran GJ, Krishnadasan A, Gorwitz RJ, et al. Methicillin-resistant S. aureus infections among patients in the emergency department. N Engl J Med 2006;355:666-74.

14. Vourli S, Vagiakou H, Ganteris G, et al. High rates of community-acquired, Panton-Valentine leukocidin (PVL) - positive methicillin-resistant $\mathrm{S}$. aureus (MRSA) infections in adult outpatients in Greece. Euro Surveill 2009;14:1-4.

15. Nathwani D, Morgan M, Masterton RG, et al. Guidelines for UK practice for the diagnosis and management of methicillinresistant Staphylococcus aureus (MRSA) infections presenting in the community. $\mathrm{J}$ Antimicrob Chemother 2008;61:976-94.

16. Klevens RM, Morrison MA, Nadle J, et al. Invasive methicillin-resistant Staphylococcus aureus infections in the United States. JAMA 2007;298:1763-71.

17. Kallen AJ, Brunkard J, Moore Z, et al. Staphylococcus aureus community-associated pneumonia during the 2006-2007. Ann Emerg Med 2009;53:358-65.

18. Popovich KJ, Weinstein RA, Hota B. Are community-associated methicillin-resistant Staphylococcus aureus (MRSA) strains 
replacing traditional nosocomial MRSA strains? Clin Infect Dis 2008;46:787-94.

19. Neofytos D, Kuhn B, Shen S, et al. Emergence of Staphylococcal Cassette Chromosome mec Type IV methicillinresistant Staphylococcus aureus as a cause of ventilator-associated pneumonia. Infect Control Hosp Epidemiol 2007;28: 1206-9.

20. Lobo LJ, Reed KD, Wunderink RG. Expanded clinical presentation of community-acquired MRSA pneumonia. Chest 2010 Available from: http://chestjournal.chestpubs.org/content/early/2010/02/17/chest.09-1562.

21. Gordon RJ, Lowy FD. Pathogenesis of methicillin-resistant Staphylococcus aureus infection. Clin Infect Dis 2008;46:S350-9.

22. Santos JWA, Nascimento DZ, Guerra VA, et al. Community-acquired staphylococcal pneumonia. J Bras Pneumol 2008;34:683-9.

23. Miller LG, Diep BA. Colonization, fomites, and virulence: Rethinking the pathogenesis of community-associated methicillinresistant Staphylococcus aureus infection. Clin Infect Dis 2008;46:752-60.

24. Murray RJ, Robinson J0, White JN, et al. Community-acquired pneumonia due to pandemic A(H1N1)2009 influenza virus and methicillin resistant Staphylococcus aureus co-infection. PLoS One 2010;5: e8705.

25. Genestier AL, Michallet MC, Prevost G, et al. Staphylococcus aureus Panton-Valentine leukocidin directly targets mitochondria and induces Bax-independent apoptosis of human neutrophils. J Clin Invest 2005;115: 3117-27.

26. Labandeira-Rey M, Couzon F, Boisset S, et al. Staphylococcus aureus PantonValentine leukocidin causes necrotizing pneumonia. Science 2007;315:1130-3.

27. Loffler B, Hussain M, Grundmeier M, et al. Staphylococcus aureus Panton-Valentine leukocidin is a very potent cytotoxic factor for human neutrophils. PLoS Pathog 2010; 6:e1000715.

28. Zhang K, McClure JA, Elsayed S, et al. Coexistence of Panton-Valentine leukocidin-positive and -negative communityassociated methicillin-resistant Staphylococcus aureus USA400 sibling strains in a large Canadian health-care region. J Infect Dis 2008;197:195-204.

29. Hamilton SM, Bryant AE, Carroll KC, et al. In vitro production of Panton-Valentine leukocidin among strains of methicillinresistant Staphylococcusaureus causing diverse infections. Clin Infect Dis 2007;45: 1550-8.

30. Gillet Y, Issartel B, Vanhems P, et al. Association between Staphylococcus aureus strains carrying gene for PantonValentine leukocidin and highly lethal necrotising pneumonia in young immuno- competent patients. Lancet 2002;359:753-9.

31. Hageman JC, Uyeki TM, Francis JS, et al. Severe community-acquired pneumonia due to Staphylococcus aureus, 2003-2004 influenza season. Emerg Infect Dis 2006;12:894-9.

32. Francis JS, Doherty MC, Lopatin U, et al. Severe community-onset pneumonia in healthy adults caused by methicillinresistant Staphylococcus aureus carrying the Panton-Valentine leukocidin genes. Clin Infect Dis 2005;40:100-7.

33. CDC. Severe methicillin-resistant Staphylococcus aureus communityacquired pneumonia associated with influenza-Louisiana and Georgia, December 2006-January 2007. Morb Mort Wkly Rep 2007;56:325-9.

34. Nguyen ET, Kanne JP, Hoang LMN, et al. Community-acquired methicillin-resistant Staphylococcus aureus pneumonia. Radiologic and computed tomography findings. J Thorac Imaging 2008;23:13-9.

35. Mandell LA, Wunderink RG, Anzueto A, et al. Infectious Disease Society of America/ American Thoracic Society consensus guidelines on the management of community-acquired pneumonia in adults. Clin Infect Dis 2007;44:S27-72.

36. Barton M, Hawkes M, Moore D, et al. Guidelines for the prevention and management of community-associated methicillin-resistant Staphylococcus aureus: A perspective for Canadian health care practitioners. Can J Infect Dis Med Microbiol 2006;17:4-24C.

37. Rubinstein E, Kollef MH, Nathwani D. Pneumonia caused by methicillin-resistant Staphylococcus aureus. Clin Infect Dis 2008;46:S378-85.

38. Stürenburg E. Rapid detection of methicillin-resistant Staphylococcus aureus directly from clinical samples: methods, effectiveness and cost considerations. Ger Med Sci 2009;6:1-19.

39. Al-Talib H, Chan YY, Al-Khateeb A, et al. A pentaplex PCR assay for the rapid detection of methicillin-resistant Staphylococcus aureus and Panton-Valentine leucocidin. BMC Microbiol 2009, 9:113.

40. Gillet Y, Vanhems P, Lina G, et al. Factors predicting mortality in necrotizing community-acquired pneumonia caused by Staphylococcus aureus containing PantonValentine leukocidin. Clin Infect Dis 2007;45:315-21.

41. Lindsay N. Community-acquired MRSA. CMAJ 2006;175:145-6.

42. Shinefield H, Black S, Fattom, et al. Use of a Staphylococcal aureus conjugate vaccine in patients receiving hemodialysis. $\mathrm{N}$ Engl J Med 2002;346:491-6.

43. Schaffer AC, Lee JC. Vaccination and passive immunisation against Staphylococcus aureus. Int J Antimicrob Agents 2008;32: S71-8.

44. Garcia-Lara J, Foster SJ. Anti-Staphylococcus aureus immunotherapy: Current status and prospects. Curr Opin Pharmacol 2009;9:552-7.

45. De Jonge M, Burchfield D, Bloom B, et al. Clinical trial of safety and efficacy of INHA21 for the prevention of nosocomial staphylococcal bloodstream infection in premature infants. J Pediatr 2007;151:260-5.

46. De Leo FR, Otto M. An antidote for Staphylococcus aureus pneumonia? J Exp Med 2008;205:271-4.

47. Brown EL, Dumitrescu 0, Thomas D, et al. The Panton-Valentine leukocidin vaccine protects mice against lung and skin infections caused by Staphylococcus aureus USA300. Clin Microbiol Infect 2009;15:15664.

48. Pea F, Viale P. The antimicrobial therapy puzzle: could pharmacokinetic-pharmacodynamic relationships be helpful in addressing the issue of appropriate pneumonia treatment in critically ill patients? Clin Infect Dis 2006;42:1764-71.

49. Rello J, Sole-Violan J, Sa-Borges M, et al. Pneumonia caused by oxacillin-resistant Staphylococcus aureus treated with glycopeptides. Crit Care Med 2005;33:1983-7.

50. Sakoulas G, Moellering RC. Increasing antibiotic resistance among methicillinresistant Staphylococcus aureus strains. Clin Infect Dis 2008;46:S360-7.

51. Moellering RC. Current treatment options for community-acquired methicillinresistant Staphylococcus aureus infection. Clin Infect Dis 2008;46:1032-7.

52. Micek ST. Alternatives to vancomycin for the treatment of methicillin-resistant Staphylococcus aureus infections. Clin Infect Dis 2007;45:S184-90.

53. Wunderlink RG, Rello J, Cammarata SK, et al. Linezolid vs vancomycin. Analysis of two double-blind studies of patients with methicillin-resistant Staphylococcus aureus nosocomial pneumonia. Chest 2003;124:1789-97.

54. Micek ST, Dunne M, Kollef MH. Pleuropulmonary complications of PantonValentine leukocidin-positive communityacquired methicillin-resistant Staphylococcus aureus. Importance of treatment with antimicrobials inhibiting exotoxin production. Chest 2005;128:2732-8.

55. Peck TE, Hill SA, Williams M. (eds) Rifamycins. In: Pharmacology for anaesthesia and intensive care. 2nd edn. London: Greenwich Medical Media Ltd, 2003, pp 325-26.

56. Proctor RA. Role of folate antagonists in the treatment of methicillin-resistant Staphylococcus aureus infection. Clin Infect Dis 2008;46:584-93. 
57. Lewis JS, Jorgensen JH. Inducible clindamycin resistance in Staphylococci: Should clinicians and microbiologists be concerned? Clin Infect Dis 2005;40:280-5.

58. Roberts JA, Lipman J. Pharmacokinetic issues for antibiotics in the critically ill patient. Crit Care Med 2009;37:840-51.

59. Silverman JA, Mortin LI, VanPraagh ADG, et al. Inhibition of daptomycin by pulmonary surfactant: in vitro modeling and clinical impact. J Infect Dis 2005;191:2149-52.

60. Rupp ME, Preston Holey H Jr, Lutz J, et al. Phase II, randomized, multicenter, doubleblind, placebo-controlled trial of a polyclonal anti-Staphylococcus aureus capsular polysaccharide immune globulin in treat- ment of Staphylococcus aureus bacteremia. Antimicrob Agents Chemother 2007;51:4249-54.

61. Gauduchon V, Cozon G, Vandenesch F, et al. Neutralization of Staphylococcus aureus Panton-Valentine leukocidin by intravenous immunoglobulin in vitro. $\mathrm{J}$ Infect Dis 2004;189:346-53. 